\title{
A rare tumor of the gallbladder: Small cell neuroendocrine carcinoma
}

\author{
(1) Nuray Kepil,, (1) Ozan Akinci, ${ }^{2}$ (i) Sebnem Batur, ${ }^{1}$ (1) A. Kagan Zengin ${ }^{3}$ \\ ${ }^{1}$ Department of Clinical Pathology, Istanbul University-Cerrahpasa, Cerrahpasa Faculty of Medicine, Istanbul, Turkey \\ 2Department of General Surgery, Hakkari State Hospital, Hakkari, Turkey \\ ${ }^{3}$ Department of General Surgery, Istanbul University-Cerrahpasa, Cerrahpasa Faculty of Medicine, Istanbul, Turkey
}

\begin{abstract}
Small cell neuroendocrine carcinoma of the gallbladder is a rare, aggressive malignancy that does not yet have a standard treatment protocol. In this case report, we aimed to share our experience about a 74-year-old male patient presented to our clinic with the complaint of episodic abdominal pain and underwent cholecystectomy under elective conditions due to a polyp with a diameter of $4 \mathrm{~cm}$ visualized in the gallbladder on ultrasound and diagnosed with neuroendocrine carcinoma as a result of histopathological examination.
\end{abstract}

Keywords: Cholecystectomy; gallbladder; neuroendocrine tumor; small cell.

Cite this article as: Kepil N, Akinci O, Batur S, Zengin AK. A rare tumor of the gallbladder: Small cell neuroendocrine carcinoma. North Clin Istanb 2020;7(6):624-627.

$\mathrm{T}$ he neuroendocrine cells are capable of secreting neurotransmitters, neuromodulators and neuropeptide hormones, located in the gastrointestinal and respiratory system mucosae and forming the largest endocrine organ of the human body. Gastroenteropancreatic neuroendocrine tumors (GEP-NET) originate from the neuroendocrine cells forming the diffuse neuroendocrine system of the gastrointestinal tract.

Primary neuroendocrine tumors of the gallbladder account for $2.2 \%$ of all gallbladder neoplasms and account for $0.2 \%$ of all NETs [1]. Neuroendocrine carcinoma of the gallbladder - small cell type (NEC-sct) is extremely rare neoplasia with an aggressive character and high malignancy potential, and it has a low survival rate.

In this case report, we presented a 74-year-old male patient whom we diagnosed with small cell neuroendocrine carcinoma of the gallbladder and who has been alive for 42 months after cholecystectomy.

\section{CASE REPORT}

A 74-year-old male patient was admitted to our clinic with the complaint of episodic pain in the right upper quadrant of the abdomen. He had a history of hypertension and 30 packs/year of smoking. There was no specific pathology with physical examination or laboratory tests. Abdominal ultrasound (US) revealed a solid mass with a diameter of $4 \mathrm{~cm}$ at the fundus of the gallbladder. A heterogeneous polypoid lesion of $4 \times 3 \times 1.5 \mathrm{~cm}$ dimensions, originating from the fundus of the gallbladder, was identified on the magnetic resonance imaging (MRI) of the upper abdomen. Radical cholecystectomy, including the liver bed $(>2 \mathrm{~cm})$, was performed. The patient was discharged on the second postoperative day. The macroscopic examination of the piece showed tumoral lesion fundus/corpus localization and ulcerated surface (Fig. 1). 


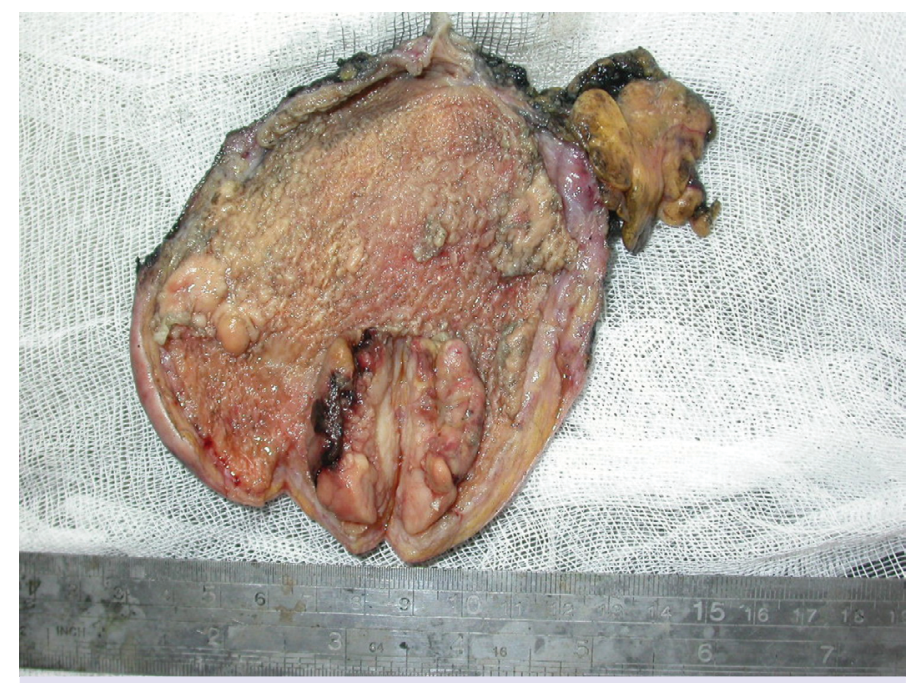

FIGURE 1. Fungating-polypoid mass with central ulceration at the fundus of the gallbladder.

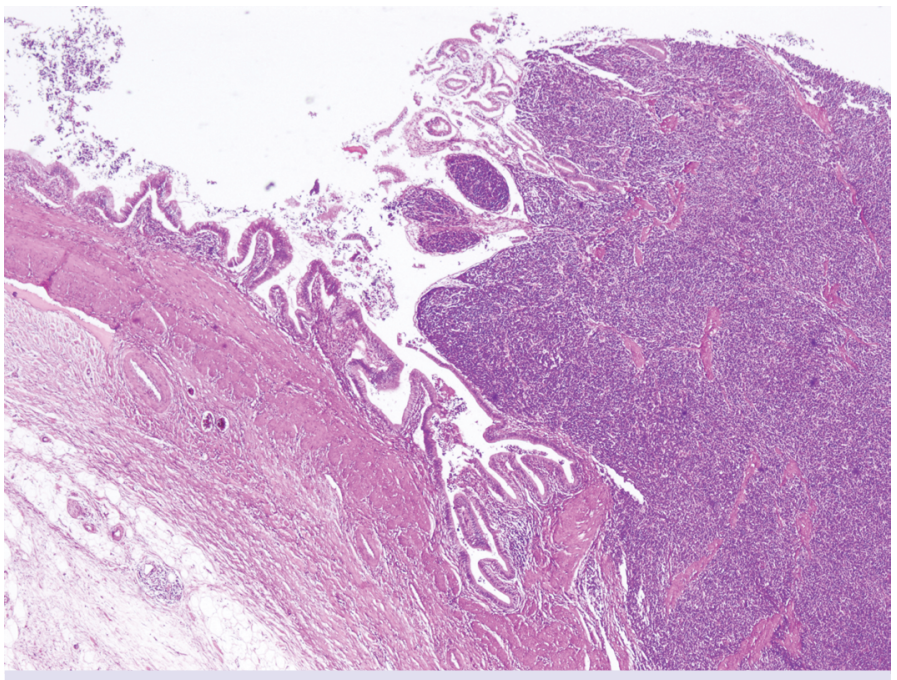

FIGURE2. $x 40, \mathrm{H} \& \mathrm{E}$; tumor in a solid pattern.

The microscopic examination showed a tumor consisting of islets of trabecular or solid pattern formed by cells with a hyperchromatic nucleus, indistinct nucleoli and narrow cytoplasm (Fig. 2, 3).

The tumor was invasive up to the serosa. Mitotic activity was high (35/10 HPF). Lymphovascular invasion and perineural invasion were present. In immunohistochemical evaluation, cytoplasmic punctate linear positive staining for pan-cytokeratin in focal areas, diffuse cytoplasmic positive staining for synaptophysin and CD56, focal weak positive staining for chromogranin A (Fig. 4) were detected.

TTF-1 (8g7g3/1) was performed immunohistochemically to eliminate the metastasis of small cell carci-

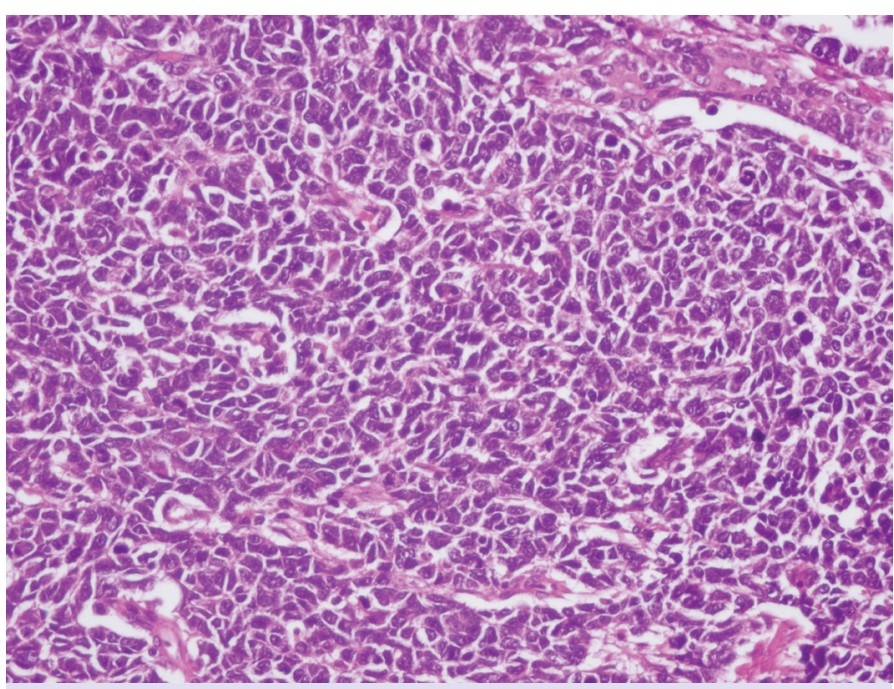

FIGURE 3. x400, H\&E; cells with hyperchromatic nucleus, indistinct nucleoli and narrow cytoplasm.

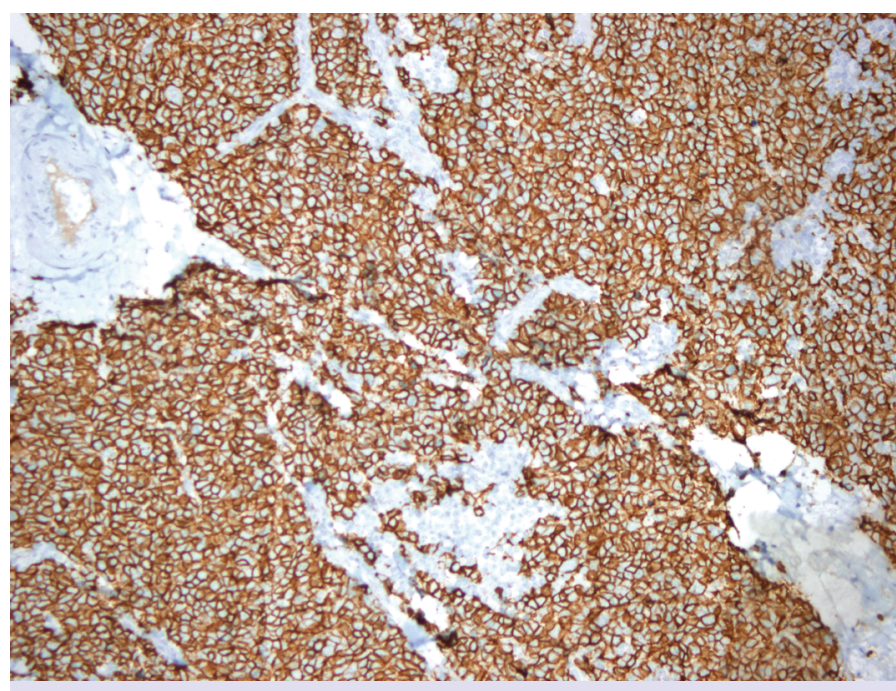

FIGURE 4. x200, synaptophysin; diffuse cytoplasmic positive reaction.

noma of the lung, and a negative result was obtained. The Ki-67 proliferative index was $80-90 \%$ (Fig. 5).

In the light of these parameters, the diagnosis of the patient was evaluated as Grade III-small cell type neuroendocrine carcinoma originating from the gallbladder. F18-fluorodeoxyglucose (FDG) Positron Emission Tomography (PET) taken on the postoperative third week showed no evidence of residual tumor or metastasis. Chemotherapy was initiated for the patient in the postoperative fourth week. A total of six cycles of etoposide $\left(100 \mathrm{mg} / \mathrm{m}^{2} /\right.$ day $)$ and cisplatin $\left(50 \mathrm{mg} / \mathrm{m}^{2} /\right.$ day $)$ were administered at 21 day intervals. There was no evidence of recurrence or me- 


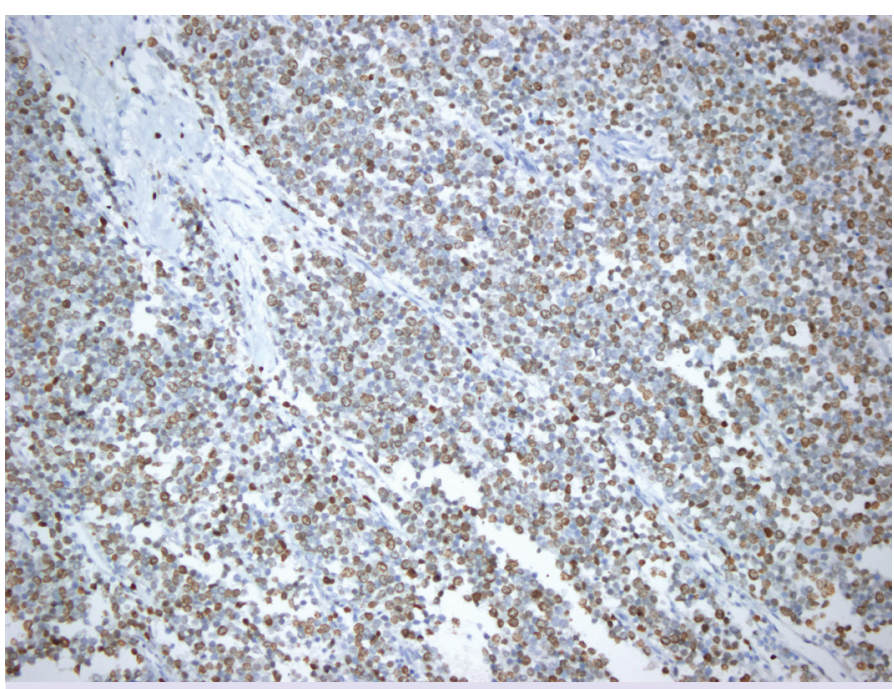

FIGURE 5. x200, The Ki- 67 proliferative index; $80-90 \%$.

tastasis on the PET scan performed after the completion of chemotherapy. Forty-two months have passed after the operation and the patient is still alive. Informed consent was obtained from the patient for this study.

\section{DISCUSSION}

Although neuroendocrine tumors may show up anywhere around the body, $67 \%$ of the cases arise in the gastrointestinal tract and $25 \%$ arise in the bronchopulmonary tract [2]. The neuroendocrine cells of the gastrointestinal tract originate from the stem cells located at the neck of the gastric glands or the base of the intestinal crypts. These cells differentiate into functionally different neuroendocrine cell types by the effects of Math1 and Neurogenin 3 transcription factors. The terminology, classification and staging of GEP-NETs have shown significant changes over the years as of WHO 1980 and have been most recently updated in WHO 2017 [3]. Our case was diagnosed in 2015 and defined as Grade III NEC - sct by being evaluated according to WHO 2010 and WHO 2017 as well.

Small cell carcinoma of the gallbladder is an extremely rare, poorly differentiated type of GEP-NET. Gallbladder NEC-sct has a worse prognosis and lower survival rate than gallbladder adenocarcinoma. According to Surveillance, Epidemiology and End Results (SEER), the 1-year survival rate of gallbladder NEC-sct is $21 \%$ and the 5-year survival rate is $0 \%$ [4]. Gallbladder NEC-sct is more common in women than in men [5]. Cholelithiasis is thought to be effective in the etiology [6].
It is difficult to diagnose gallbladder NEC-sct using US, CT, or MRI. The diagnosis is usually made incidentally with the examination of pieces of cholecystectomy performed due to cholelithiasis or polyps. The studies conducted have shown that PET/CT has high sensitivity in diagnosing gallbladder NEC-sct [7].

In a literature review, Adachi et al. [6] reported that $80 \%$ of the cases were pure NEC-sct and $20 \%$ were combined NEC-sct histopathologically. Immunohistochemically, gallbladder NEC-sct is known to express neuroendocrine markers, such as synaptophysin, chromogranin $\mathrm{A}$, and CD56. Contrary to NETs in which neuroendocrine markers are diffuse positive, gallbladder NEC-sct shows more focal staining. According to the literature, chromogranin A antibody gives a weak positive reaction as the differentiation decreases [8]. Some of the GEPNETs express chromogranin $B$, thus producing a weak positive reaction with chromogranin $\mathrm{A}$, whereas producing a strong positive reaction with synaptophysin antibody regardless of differentiation [9].

The surgical treatment for gallbladder NEC-sct is still the only curative treatment option. The surgical procedures, such as radical cholecystectomy, extended cholecystectomy, including regional lymph node dissection and hepatic lobectomy, are among these options. Additional treatment modalities such as chemotherapy or radiotherapy may be needed for gallbladder NEC-sct cases with a low likelihood of curative surgery due to poor prognosis. There are studies suggesting that the combined use of chemotherapeutic agents, such as cisplatin, etoposide, 5-fluorouracil, gemcitabine and streptozocin, are useful $[10,11]$.

Gallbladder NEC-sct has a very low incidence and poor prognosis. Early diagnosis and aggressive treatment, including radical resection and chemoradiation, have been reported to produce the best clinical outcomes. In this case report, we performed radical cholecystectomy and adjuvant chemotherapy in a patient with gallbladder NEC-sct. The patient is still alive forty-two months after the operation. We hope that further progress in multimodal therapy will improve the survival rates of gallbladder NEC-sct.

\section{Conclusion}

Small-cell neuroendocrine carcinoma is one of the rare tumors of the gallbladder and should be kept in mind in the differential diagnosis of poorly differentiated malignant tumors. The possibility of metastasis from other organs 
should be excluded before it is accepted to be the primary tumor of the gallbladder. In some cases, radical surgery with chemotherapy or radiation therapy could increase the survival rate of patients. If future studies focus on understanding the molecular biology of this tumor, more effective treatment modalities may be able to develop.

Authorship Contributions: Concept - OA; Design - NK; Supervision - SB; Materials - NK; Data collection and/or processing - OA; Analysis and/or interpretation - NK; Literature review - OA; Writing - OA; Critical review - AKZ.

Informed Consent: Written informed consent was obtained from the patient for the publication of the case report and the accompanying images.

Conflict of Interest: No conflict of interest was declared by the authors.

Financial Disclosure: The authors declared that this study has received no financial support.

\section{REFERENCES}

1. Mezi S, Petrozza V, Schillaci O, La Torre V, Cimadon B, Leopizzi M, et al. Neuroendocrine tumors of the gallbladder: a case report and review of the literature. J Med Case Rep 2011;5:334. [CrossRef]
2. Modlin IM, Lye KD, Kidd M. A 5-decade analysis of 13,715 carcinoid tumors. Cancer 2003;97:934-59. [CrossRef]

3. Lloyd RV, Osamura RY, Klöppel G, Rosal J. WHO classification of tumors of endocrine organs. $4^{\text {th }}$ ed. 2017. Available at: https://www.iarc. $\mathrm{fr} /$ news-events/who-classification-of-tumours-of-endocrine-organs/. Accessed Oct 05, 2020.

4. Eltawil KM, Gustafsson BI, Kidd M, Modlin IM. Neuroendocrine tumors of the gallbladder: an evaluation and reassessment of management strategy. J Clin Gastroenterol 2010;44:687-95. [CrossRef]

5. Moskal TL, Zhang PJ, Nava HR. Small cell carcinoma of the gallbladder. J Surg Oncol 1999;70:54-9. [CrossRef]

6. Adachi T, Haraguchi M, Irie J, Yoshimoto T, Uehara R, Ito S, et al. Gallbladder small cell carcinoma: a case report and literature review. Surg Case Rep 2016;2:71. [CrossRef]

7. Kim DM, Yang SO, Han HY, Kim KS, Son HJ. Small Cell Carcinoma of the Gallbladder: (18)F-FDG PET/CT Imaging Features-A Case Report. Nucl Med Mol Imaging 2010;44:213-6. [CrossRef]

8. Taupenot L, Harper KL, O'Connor DT. The chromogranin-secretogranin family. N Engl J Med 2003;348:1134-49. [CrossRef]

9. Chetty R. An overview of practical issues in the diagnosis of gastroenteropancreatic neuroendocrine pathology. Arch Pathol Lab Med 2008;132:1285-9.

10. Mahipal A, Gupta S. Small-cell carcinoma of the gallbladder: report of a case and literature review. Gastrointest Cancer Res 2011;4:135-6.

11. Bahadur S, Shaukat A, Gibbs J, Litwin A, Nava H, Melnyk M, Javle M. Cisplatin and gemcitabine for small cell carcinoma of the gall bladder. Am J Clin Oncol 2005;28:425-6. [CrossRef] 